\title{
Prevalence of Anal and Oral HPV DNA and HPV Seroprevalence among Transgender Women in Two Cities, United States, 2012-2014
}

Vidisha Singh, MSPH; ${ }^{1}$ Beau Gratzer, MPP;2 Pamina M. Gorbach, MHS, DrPH; ${ }^{3}$ Richard A. Crosby, PhD;4 Gitika Panicker, PhD;5 Martin Steinau, PhD; ${ }^{5}$ Elizabeth R. Unger, PhD, MD; ${ }^{5}$ Lauri E. Markowitz, MD; ${ }^{1}$ Elissa Meites, MD, MPH ${ }^{1}$

1. Division of Viral Diseases, National Center for Immunization and Respiratory Diseases, Centers for Disease Control and Prevention, Atlanta, GA, USA; 2. Howard Brown Health, Chicago, IL, USA; 3. Department of Epidemiology, Fielding School of Public Health, University of California at Los Angeles, Los Angeles, CA, USA 4. College of Public Health, University of Kentucky at Lexington, Lexington, KY, USA; 5. Division of High-Consequence Pathogens and Pathology, National Center for Emerging and Zoonotic Infectious Diseases, Centers for Disease Control and Prevention, Atlanta, GA, USA

Background: Human papillomavirus (HPV) is the most common sexually transmitted infection. Prevalence is particularly high among men who have sex with men (MSM), yet little is known about prevalence among transgender women.

Objective: We assessed evidence of HPV among a convenience sample of transgender women compared with cisgender men.

Methods: This was a cross-sectional study of 1,033 gay, bisexual, and other MSM assigned male sex at birth aged 18-26 years from two lesbian, gay, bisexual and transgender (LGBT) community health clinics in Chicago, IL and Los Angeles, CA during 2012-2014. Data was collected by computer-assisted self-interview about demographics, gender identity, vaccinations, HIV status, and other healthcare services; self-collected anal and oral specimens were tested for HPV DNA (37 types); serum were tested for antibodies to quadrivalent HPV vaccine types $(6,11,16,18)$. Frequencies, proportions, prevalence ratios (PRs), 95\% confidence intervals (Cls) were calculated using SAS.
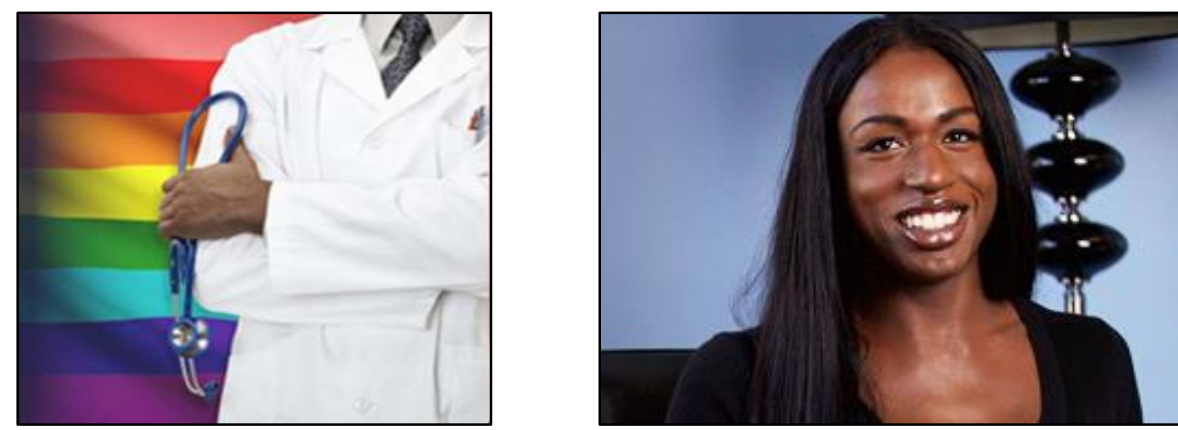

Table 1. Characteristics of Participating Transgender Women $(n=49)$ Characteristic $n \quad(\%)$

Age, years

$18-21$

$22-26$

18 (36.7)

Race/ethnicity

Non-Hispanic white

Non-Hispanic black

Non-Hispanic Asian/Pacific Islander

Hispanic/Latino (any race)

Other/unknown

Education

High school or below

Some college/2-year or technical degree

4-year degree or equivalent/higher

Other/unknown

31 (63.3)

ealth insurance status

Insured

None or unknown

(0.0)

30 (61.2)

1 (2.0)

8 (16.3)

10 (20.4)

35 (71.4)

$7 \quad(14.3)$

4 (8.2)

3 (6.1)

22 (44.9)

27 (55.1)

Sexual orientation

Gay/homosexual

Bisexual

Heterosexual/straight

Other/unknown

19 (38.8)

14 (28.6)

$8 \quad(16.3)$

8 (16.3)

Number of lifetime partners, male or female

0-5

6-10

18 (36.7)

8 (16.3)

7 (14.3)

$11-20$

$>20$

Other/unknown

12 (24.5)

HIV status, self-reported

Positive

13 (26.5)

Negative or unknown

36 (73.5)

Visited healthcare provider in past 12 months Yes

No or unknown

39 (79.6)

$10(20.4)$

Ever received any HPV vaccination Yes
Results: Demographic characteristics were assessed among all 49 transgender women: Most were aged 22-26 years (63.3\%), nonHispanic black (61.2\%), identified as either gay/homosexual or bisexual (65.4\%), and had $\leq 10$ lifetime sex partners (53.1\%). A small proportion were HIV-positive (26.5\%); most had visited a healthcare provider in the past 12 months (79.6\%); few received any HPV vaccination (10.2\%).

HPV prevalence and seroprevalence were compared for 44 unvaccinated transgender women and 855 unvaccinated cisgender men: Among unvaccinated transgender women, anal HPV prevalence (88.6\%) and seroprevalence (84.1\%) were high. In anal specimens, prevalence of any HPV DNA was greater among unvaccinated transgender women compared with cisgender men (PR: 1.3), and also for $\geq 1$ vaccine type (PR: 1.4). In oral specimens, HPV DNA prevalence was not significantly different between groups. Detection of antibodies to $\geq 1$ vaccine type was greater among transgender women compared with cisgender men (PR: 1.5). Most transgender women $(65.9 \%)$ and cisgender men $(90.6 \%)$ were naïve to $\geq 1$ quadrivalent vaccine type. Differences were maintained in analyses limited to HIVnegative transgender women and cisgender men.

\begin{tabular}{|c|c|c|c|c|}
\hline \multicolumn{5}{|c|}{$\begin{array}{l}\text { Table 2. HPV Prevalence in Unvaccinated Transgender Women } \\
(n=44) \text { and Cisgender Men }(n=855)\end{array}$} \\
\hline & $\begin{array}{c}\text { Unvaccinated } \\
\text { Transgender } \\
\text { Women }\end{array}$ & \multicolumn{2}{|c|}{$\begin{array}{l}\text { Unvaccinated } \\
\text { Cisgender Men }\end{array}$} & \multirow[b]{2}{*}{ PR $(95 \% \mathrm{Cl})$} \\
\hline & $\mathrm{n} \quad(\%)$ & $\mathrm{n}$ & $(\%)$ & \\
\hline \multicolumn{5}{|l|}{ Anal HPV } \\
\hline Any HPV & $39(88.6)$ & 606 & $(70.9)$ & $1.3(1.2,1.4)$ \\
\hline$\geq 1$ Vaccine Type & $22(50.0)$ & 311 & (36.4) & $1.4(1.0,1.9)$ \\
\hline \multicolumn{5}{|l|}{ Oral HPV } \\
\hline Any HPV & $4 \quad(9.1)$ & 81 & (9.5) & $1.0(0.4,2.5)$ \\
\hline$\geq 1$ Vaccine Type & $1 \quad(2.3)$ & 26 & (3.0) & $0.7(0.1,5.4)$ \\
\hline Antibody $\geq 1$ Vaccine & & & & \\
\hline $\begin{array}{l}\text { Type } \\
\text { Naïve to } \geq 1 \text { Vaccine }\end{array}$ & 37 (84.1) & 467 & $(54.6)$ & $1.5(1.3,1.8)$ \\
\hline Typet & $29(65.9)$ & 775 & $(90.6)$ & $0.7(0.6,0.9)$ \\
\hline
\end{tabular}

Limitations: Enrollment of participants at community LGBT clinics represents those more likely to visit healthcare providersSelf-reported HPV vaccination might underestimate the true number of vaccinated participants The proportion of participants naïve to $\geq 1$ vaccine type would likely be higher for 9-valent HPV vaccine than for quadrivalent vaccine.

Conclusions: HPV was common among transgender women. Anal prevalence and seroprevalence were significantly higher among transgender women compared with cisgender men in this study. Participants not previously exposed to all vaccine types could benefit from vaccination through catch-up age. Although vaccination ideally occurs prior to HPV exposure, these findings support current national recommendations to vaccinate transgender people and MSM through age 26 years.

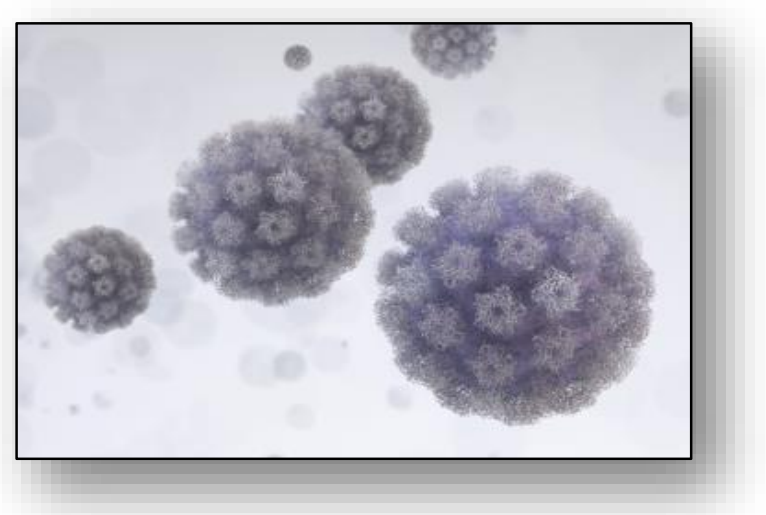

